EPJ Web of Conferences 59, 13013 (2013)

DOI: $10.1051 /$ epjconf/20135913013

(C) Owned by the authors, published by EDP Sciences, 2013

\title{
Absolute measurement of the DT primary neutron yield on the National Ignition Facility
}

\author{
R.J. Leeper ${ }^{1, a}$, D.L. Bleuel ${ }^{2}$, J.A. Frenje ${ }^{3}$, M.J. Eckart ${ }^{2}$, E. Hartouni², \\ J.D. Kilkenny ${ }^{4}$, D.T. Casey ${ }^{3}$, G.A. Chandler ${ }^{1}$, G.W. Cooper ${ }^{5}$, V.Yu. Glebov ${ }^{6}$, \\ C. Hagmann ${ }^{2}$, M. Gatu Johnson ${ }^{3}$, J.P. Knauer ${ }^{6}$, K.M. Knittel ${ }^{2}$, \\ and F.H. Seguin ${ }^{3}$ \\ 1 Sandia National Laboratories, Albuquerque, NM 87185, USA \\ 2 Lawrence Livermore National Laboratory, Livermore, CA 94550, USA \\ ${ }^{3}$ Massachusetts Institute of Technology, Cambridge, MA 02139, USA \\ 4 General Atomics, San Diego, CA 92186, USA \\ 5 University of New Mexico, Albuquerque, NM 87131, USA \\ 6 University of Rochester, Rochester, NY 14627, USA \\ 7 State University of New York-Geneseo, Geneseo, NY 14454, USA
} L.A. Linden-Levy², S.J. Padalino ${ }^{7}$, R.D. Petrasso ${ }^{3}$, C.L. Ruiz ${ }^{1}$, T.C. Sangster ${ }^{6}$

\begin{abstract}
The measurement of the absolute neutron yield produced in inertial confinement fusion target experiments conducted on the National Ignition Facility (NIF) is essential in benchmarking progress towards the goal of achieving ignition on this facility. This paper describes three independent diagnostic techniques that have been developed to make accurate and precise DT neutron yield measurements on the NIF.
\end{abstract}

\section{INTRODUCTION}

Three diagnostic systems have been developed to perform measurements of the absolute DT primary neutron yield on the NIF. An accurate and precise measurement of neutron yield to $\sim 5 \%$ is essential to gauge progress towards ignition on NIF but several factors, especially neutron scattering cause errors. The first diagnostic is the magnetic recoil spectrometer that magnetically analyzes deuterium ions generated from neutron elastic scattering in a thin CD foil. The second system is a copper activation system that employs two different $\mathrm{Cu}$ samples. The third system is a zirconium activation system that employs three different $\mathrm{Zr}$ samples. The philosophy used in developing these three yield diagnostics is to use physical processes that are independent of each other to minimize systematic errors inherent in each technique. The focus of the paper will be on identifying and estimating these systematic errors as well as addressing statistical errors as a function of total neutron yield for each of these diagnostics. Finally, the paper will discuss a standard weighted least-squares procedure of combining the measured yields and associated errors of each technique into a final yield and best-estimated error. This procedure is an adaptation of the procedure used in particle physics to combine measurements and associated errors from a number of different experiments into a best-estimated value and corresponding error.

\footnotetext{
ae-mail: rjleepe@sandia.gov
}

This is an Open Access article distributed under the terms of the Creative Commons Attribution License 2.0, which permits unrestricted use, distribution, and reproduction in any medium, provided the original work is properly cited. 
EPJ Web of Conferences

Table 1. Systematic uncertainties for the MRS diagnostic are shown here.

\begin{tabular}{|c|c|c|c|c|}
\hline MRS Parameter & Absolute & High-Res (\%) & Med-Res (\%) & Low-Res (\%) \\
\hline Foil area uncertainty & $\pm 0.2 \mathrm{~cm}^{2}$ & \pm 1.5 & \pm 1.5 & \pm 1.5 \\
\hline Foil distance uncertainty & $\pm 0.3 \mathrm{~cm}$ & \pm 1.1 & \pm 1.1 & \pm 1.1 \\
\hline Number density uncertainty & $\pm 10^{21} \mathrm{~cm}^{3}$ & \pm 1.3 & \pm 1.3 & \pm 1.3 \\
\hline Foil thickness uncertainty & $\pm 2.0 \mu \mathrm{m}$ & \pm 2.0 & \pm 0.8 & \pm 0.4 \\
\hline ND-cross section uncertainty & $\pm 12 \mathrm{mb} / \mathrm{sr}$ & \pm 2.3 & \pm 2.3 & \pm 2.3 \\
\hline Magnet aperture area uncertainty & $\pm 0.2 \mathrm{~cm}^{2}$ & \pm 1.0 & \pm 1.0 & \pm 1.0 \\
\hline Magnet aperture distance uncertainty & $\pm 0.1 \mathrm{~cm}$ & \pm 0.02 & \pm 0.02 & \pm 0.02 \\
\hline Total systematic uncertainty for yield & & \pm 4.4 & \pm 4.0 & \pm 3.9 \\
\hline
\end{tabular}

\section{NIF ABSOLUTE NEUTRON MEASUREMENTS}

One approach developed to measure the absolute DT primary yield on NIF is to use the NIF Magnetic Recoil Spectrometer (MRS). A detailed description of this diagnostic is given in Ref. [1-3]. Briefly the MRS has three main components. The first component is a CD foil positioned $26 \mathrm{~cm}$ from the implosion for production of recoil deuterons from incident neutrons; a focusing magnet located outside the NIF target chamber for energy dispersion and focusing of forward-scattered recoil deuterons onto the focal plane of the spectrometer; and an array of CR-39 nuclear track detectors, positioned at the focal plane, which records the position of each recoil particle with a detection efficiency of $100 \%$. The spectrum of the recoil deuterons is determined by position at the detector plane, and used to determine the neutron spectrum. The yield reported from the MRS diagnostic is defined as the integral of the neutron spectrum between $13 \mathrm{MeV}$ and $15 \mathrm{MeV}$ and is referred to as the NIF primary neutron yield. The NIF MRS is designed to be operated in three resolution modes: (1) high resolution for DT neutron yields in the range of $10^{15}-10^{19}$, (2) medium resolution for neutron yields in the range of $10^{14}-10^{18}$, and low resolution for neutron yields less than $10^{14}$. Table 1 shows the systematic uncertainties for the various MRS parameters and their contribution to the total systematic yield error for the MRS highresolution, medium-resolution, and low-resolution modes. Typical MRS statistical errors are small. For example, the statistical error is less than $1 \%$ at a neutron yield at $\sim 2.5 \times 10^{14}$ when operated in the medium-resolution mode.

A second measurement of the NIF absolute DT total neutron yield is performed using a copper nuclear activation detector denoted $\mathrm{Cu}$ NAD. This technique employs the ${ }^{63} \mathrm{Cu}(\mathrm{n}, 2 \mathrm{n}){ }^{62} \mathrm{Cu}\left(\beta^{+}\right)$reaction that has a total cross-section of $454.6 \mathrm{mb}$ at $14.1 \mathrm{MeV}$ [4-6]. The threshold energy for this reaction is 11.0 MeV that leads to yields inferred from $\mathrm{Cu}$ NAD that are higher by $2 \%$ to $5 \%$ relative to MRS yields depending on the neutron spectrum. The $\beta^{+}$annihilation $0.511 \mathrm{MeV}$ gamma rays are counted in $\mathrm{NaI}$ scintillator coincidence counting system that employs two $15.24 \mathrm{~cm}$ diameter by $7.62 \mathrm{~cm} \mathrm{NaI}$ detectors. The half-life of ${ }^{62} \mathrm{Cu}$ is 9.67 minutes. A thin copper sample (Cu NAD19) that is $5.08 \mathrm{~cm}$ diameter by $0.1 \mathrm{~cm}$ thick is fielded at a position $19.0 \mathrm{~m}$ from target chamber center. A second thick copper sample (Cu NAD29) that is $7.62 \mathrm{~cm}$ diameter by $0.95 \mathrm{~cm}$ thick that is fielded at a distance of $29 \mathrm{~m}$ from target chamber center. Because the ${ }^{62} \mathrm{Cu} \beta^{+}$decay has an endpoint energy of $2.9 \mathrm{MeV}$, the thin copper sample is counted sandwiched between two copper disks that are each $5.08 \mathrm{~cm}$ diameter by $0.2 \mathrm{~cm}$ thick to insure that the $\beta^{+}$stop and annihilate in a well defined region located between the two $\mathrm{NaI}$ scintillation detectors. The total systematic and typical statistical errors for the Cu NAD19 detector are shown in Table 2. Cu NAD29 views the neutron source through a rather complex neutron time-offlight system located at $22 \mathrm{~m}$. Because of the difficulty of accurately determining the neutron attenuation factor through this detector system, the Cu NAD29 detector is normalized to the Cu NAD19 detector using data obtain on several NIF neutron calibration shots. 
IFSA 2011

Table 2. Systematic and typical statistical errors for the NIF Cu NAD19 detector are shown here.

\begin{tabular}{|l|c|c|c|}
\hline Quantity & Value & Relative Error & Relative Error (\%) \\
\hline${ }^{63} \mathrm{Cu}$ Natural Abundance & 0.6917 & \pm 0.0003 & \pm 0.04 \\
\hline${ }^{62} \mathrm{Cu}$ Decay Branching Ratio & 0.9743 & \pm 0.0002 & \pm 0.02 \\
\hline Detector Counting Efficiency & 0.1445 & \pm 0.00448 & \pm 3.1 \\
\hline Self Attenuation for 511 keV $\gamma$ 's & 0.95 & \pm 0.0475 & \pm 5.0 \\
\hline Cross Section at 14.1 MeV $\left(\mathrm{cm}^{2}\right)$ & $4.5459 \mathrm{E}-25$ & $\pm 5.8642 \mathrm{E}-27$ & \pm 1.3 \\
\hline${ }^{62} \mathrm{Cu}$ Decay Half-life $(\mathrm{min})$ & 9.673 & \pm 0.008 & \pm 0.08 \\
\hline${ }^{62} \mathrm{Cu}$ Mean Half-life $(\mathrm{min})$ & 13.955 & \pm 0.011 & \pm 0.08 \\
\hline Sample Distance $(\mathrm{cm})$ & 1900.5 & \pm 1.9005 & \pm 0.1 \\
\hline Attenuation Factor & 1.965 & \pm 0.0786 & \pm 4.0 \\
\hline Total Systematic Error & & & \pm 7.3 \\
\hline Statistical Error (Yield $\left.>10^{14}\right)$ & & & $\pm 1.0-2.0$ \\
\hline
\end{tabular}

Table 3. Systematic and typical statistical errors for the NIF Zr NAD diagnostic are shown here.

\begin{tabular}{|l|c|c|}
\hline Quantity & Effect on Activity & Systematic Error (\%) \\
\hline Detector efficiency & & $\pm 2.0-5.0$ \\
\hline Gamma-ray self-shielding & $-15 \%$ & Included in detector efficiency \\
\hline Neutron "depletion" in zirconium & & \pm 0.6 \\
\hline Self-shielding differential from neutron depletion & $-3.1 \%$ & \pm 2.0 \\
\hline Cross-section uncertainty & & \pm 1.0 \\
\hline Position uncertainty & & \pm 1.1 \\
\hline Scatter/absorption off Al shield/well & $-5.7 \%$ & \pm 1.1 \\
\hline Chamber/wall scatter & & $\pm<1$ \\
\hline Scatter off nearby materials & & $\pm<1$ \\
\hline Down-scattered/non-primary neutrons & & \pm 2.0 \\
\hline Drift velocity/temperature peak shift $(<100 \mathrm{keV})$ & & \pm 2.0 \\
\hline Ion temperature peak broadening & & $\pm<1$ \\
\hline Sample purity (98.7\%) & $-1.35 \%$ & \pm 0.2 \\
\hline Sample weight/oxidation/contamination & & $\pm<1$ \\
\hline Total systematic error & & \pm 6.0 \\
\hline Statistical error (Yield $\left.>10^{14}\right)$ & & \pm 1.0 \\
\hline
\end{tabular}

A third measurement of the NIF absolute DT total neutron yield is performed using three samples of zirconium. A detailed paper on this technique is included in these proceedings [7]. Briefly, this technique uses the ${ }^{90} \mathrm{Zr}(\mathrm{n}, 2 \mathrm{n}){ }^{89} \mathrm{Zr}$ reaction that has a total cross-section of $622.0 \mathrm{mb}$ at $14.1 \mathrm{MeV}$. The threshold energy for this reaction is $12.1 \mathrm{MeV}$ which leads to yields from $\mathrm{Zr}$ activation that are typically higher by at most $1 \%$ relative to MRS yields. ${ }^{89} \mathrm{Zr}$ decays to ${ }^{89 \mathrm{~m}} \mathrm{Y}$ meta-stable state. ${ }^{89 \mathrm{~m}} \mathrm{Y}$ has a half-life of 15.663 seconds and is in equilibrium with ${ }^{89} \mathrm{Zr}$. The branching ratio of the internal transition $909 \mathrm{keV}$ gamma ray is $99.2 \%$ and the half-life of ${ }^{89} \mathrm{Zr}$ is 3.267 days. An array of high purity Ge detectors is used to count the $909 \mathrm{keV}$ lines emitted by the samples. The total systematic and typical statistical errors for this detector are shown in Table 3. 
EPJ Web of Conferences

Table 4. Comparison of yield values obtained via Cu NADs, Zr NADs, and MRS along with the NIF authorized value for a representative number of NIF shots.

\begin{tabular}{|c|c|c|c|c|c|}
\hline NIF Shot Number & $\begin{array}{l}\text { Thin } \mathrm{Cu} \\
\text { Yield }\end{array}$ & $\begin{array}{l}\text { Thick } \mathrm{Cu} \\
\text { Yield }\end{array}$ & Zr Yield & MRS Yield & $\begin{array}{l}\text { Authorized } \\
\text { NIF Value* }\end{array}$ \\
\hline $\begin{array}{c}\text { N110121 } \\
\text { Layered THD }\end{array}$ & $\begin{array}{c}2.21 \times 10^{13} \\
\pm 9.6 \%\end{array}$ & $\begin{array}{c}2.01 \times 10^{13} \\
\pm 10.0 \%\end{array}$ & $\begin{array}{c}2.08 \times 10^{13} \\
\pm 7.2 \%\end{array}$ & $\begin{array}{c}2.10 \times 10^{13} \\
\pm 5.0 \%\end{array}$ & $\begin{array}{l}2.10 \times 10^{13} \\
\pm 2.3 \%\end{array}$ \\
\hline $\begin{array}{c}\text { N110212 } \\
\text { Layered THD }\end{array}$ & $\begin{array}{c}1.27 \times 10^{14} \\
\pm 7.9 \%\end{array}$ & $\begin{array}{c}1.23 \times 10^{14} \\
\pm 7.7 \%\end{array}$ & $\begin{array}{c}1.18 \times 10^{14} \\
\pm 7.7 \%\end{array}$ & $\begin{array}{c}1.23 \times 10^{14} \\
\pm 4.5 \%\end{array}$ & $\begin{array}{c}1.25 \times 10^{14} \\
\pm 2.3 \%\end{array}$ \\
\hline $\begin{array}{c}\text { N110217 } \\
\text { Exploding Pusher }\end{array}$ & $\begin{array}{c}1.88 \times 10^{14} \\
\pm 7.9 \%\end{array}$ & $\begin{array}{l}1.95 \times 10^{14} \\
\pm 7.7 \%\end{array}$ & $\begin{array}{c}1.94 \times 10^{14} \\
\pm 7.1 \%\end{array}$ & $\begin{array}{c}1.98 \times 10^{14} \\
\pm 5.0 \%\end{array}$ & $\begin{array}{l}1.94 \times 10^{14} \\
\pm 1.5 \%\end{array}$ \\
\hline $\begin{array}{c}\text { N110603-2 } \\
\text { Exploding Pusher }\end{array}$ & $\begin{array}{l}2.18 \times 10^{14} \\
\quad \pm 7.5 \%\end{array}$ & $\begin{array}{l}2.09 \times 10^{14} \\
\pm 7.5 \%\end{array}$ & $\begin{array}{c}2.14 \times 10^{14} \\
\pm 7.4 \%\end{array}$ & $\begin{array}{c}2.21 \times 10^{14} \\
\pm 4.4 \%\end{array}$ & $\begin{array}{l}2.17 \times 10^{14} \\
\quad \pm 1.8 \%\end{array}$ \\
\hline
\end{tabular}

*Authorized values for layered shots include neutron time-of-flight detectors that were normalized to $\mathrm{Zr}$ activation.

\section{NIF NEUTRON YIELD EXPERIMENTAL RESULTS}

Table 4 shows a comparison of absolute DT yield values obtained from the $\mathrm{Cu}$ NAD19 (Thin Cu), $\mathrm{Cu}$ NAD29 (Thick $\mathrm{Cu}$ ), Zr NAD and MRS diagnostics for a representative number of NIF shots. The authorized NIF values and associated errors shown in the table are determined from all four diagnostic measurements using a standard weighted least-squares technique. This procedure is an adaptation of the procedure used in particle physics to combine measurements and associated errors from a number of different experiments into a best-estimated value and corresponding error [8]. Examination of this table shows that the absolute neutron yields determined from all four measurements are in excellent agreement to within their errors giving confidence in this set of four absolute neutron yields. Applying the weighted least square-technique to this data enables a best-estimated NIF authorized value for the absolute neutron yield and its error for each NIF shot as shown in the table.

This work performed under the auspices of the U.S. Department of Energy by Sandia, Los Alamos, and Lawrence Livermore National Laboratories under contracts DE-AC04-94AL85000, DE-AC52-06NA25396 and DE-AC52$07 N A 27344$.

\section{References}

[1] J. A. Frenje et al., Rev. Sci. Instrum. 79, 10E502 (2008)

[2] J. A. Frenje et al., Phys. Plasmas 17, 056311 (2010)

[3] J. A. Frenje et al., these proceedings

[4] R. J. Leeper, Proc. NATO Advanced Study Institute on Fast Electrical and Optical Diagnostic Principles and Techniques, Vol. 1., eds. J. E. Thompson and L. H. Luessen (Martinus Nijhoff, The Netherlands, 1986) pp. 263-315

[5] R. J. Leeper, K. H. Kim, D. E. Hebron, N. D. Wing, Nucl. Instrum. Meth. B24/25, 695 (1987)

[6] G. W. Cooper, C. L. Ruiz, Rev. Sci. Instrum. 72, 814 (2001)

[7] D. L. Bleuel et al., these proceedings

[8] K. Nakamura et al. (Particle Data Group), J. Phys. G37, 075021 (2010) 